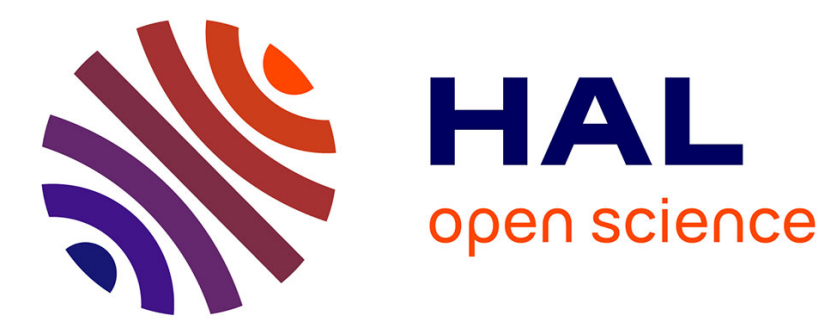

\title{
INVESTIGATION OF INNER AND OUTER SUBSHELLS VIA TRANSFER REACTIONS
}

\author{
S. Gales
}

\section{To cite this version:}

S. Gales. INVESTIGATION OF INNER AND OUTER SUBSHELLS VIA TRANSFER REACTIONS. International Symposium on Highly Excited States and Nuclear Structure Hesans 83, 1983, Orsay, France. pp.C4-39-C4-55, 10.1051/jphyscol:1984405 . jpa-00224070

\section{HAL Id: jpa-00224070 https://hal.science/jpa-00224070}

Submitted on 1 Jan 1984

HAL is a multi-disciplinary open access archive for the deposit and dissemination of scientific research documents, whether they are published or not. The documents may come from teaching and research institutions in France or abroad, or from public or private research centers.
L'archive ouverte pluridisciplinaire HAL, est destinée au dépôt et à la diffusion de documents scientifiques de niveau recherche, publiés ou non, émanant des établissements d'enseignement et de recherche français ou étrangers, des laboratoires publics ou privés. 


\title{
INVESTIGATION OF INNER AND OUTER SUBSHELLS VIA TRANSFER REACTIONS
}

\author{
S. Gales \\ Institut de Physique Nucléaire, B.P. n¹, 91406 Orsay Cédex, France
}

Résumé - Les réactions de transfert de 1 et 2 nucléons à haute énergie incidente (100 à $300 \mathrm{MeV}$ environ) ont permis d'étendre considérablement notre connaissance de la fonction de réponse du noyau à une excitation simple. Ces réactions sont particulièrement bien adaptées à l'étude des couches internes et externes de haut moment orbital.

Ces propriétés seront illustrées par quelques exemples significatifs. Sélectivité en moment orbital transféré, détermination du spin $\mathrm{J}$ des couches internes au moyen de faisceaux de particules polarisées dans le cas du pick up profond d'un nucléon dans les noyaux lourds. Nouvelles approches dans l'étude des distributions de 7 a force à deux particules : premiers résultats concernant la réaction $\left(a,{ }^{6} \mathrm{He}\right)$ à $218 \mathrm{MeV}$ d'énergie incidente.

On insistera particulièrement sur la mise en évidence récente à haute énergie d'excitation (5 à $15 \mathrm{MeV})$ d'états à une particule au moyen de réaction de stripping $(\alpha, t),\left({ }^{3} \mathrm{He}, \mathrm{d}\right)$ et $\left(\alpha,{ }^{3} \mathrm{He}\right)$. A partir de ces exemples les résultats expérimentaux seront comparés aux prédictions des modèles nucléaires qui tiennent compte explicitement du couplage entre degrés de liberté individuels et collectifs pour expliquer la fragmentation et l'étalement des forces à une particule.

Abstract - Transfer reactions, investigated a high incident energy (from 100 to $300 \mathrm{MeV}$ ), have considerably increased our knowledge of the nuclear response function to a particular simple mode of excitation of the nucleus. These reactions are particularly well suited to the study of high spin inner and outer subshells. The advantages of such approach are illustrated using few characteristic examples.

a) Strong selectivity in angular momentum transfer, determination of the spin $J$ of the inner hole state using polarized protonbeam in the case of one nucleon pick-up reaction.

b) New experimental results via the $\left(\alpha,{ }^{6} \mathrm{He}\right)$ reaction at $218 \mathrm{MeV}$ incident energy are obtained on the two-nucleon strength distributions in medium-heavy nuclei.

c) First evidence of proton and neutron single-particle strengths at high excitation energy ( 5 to $15 \mathrm{MeV}$ ) in heavy nuclei are reported using the $(\alpha, t)$ $\left(\alpha,{ }^{3} \mathrm{He}\right)$ and $\left({ }^{3} \mathrm{He}, \mathrm{d}\right)$ stripping reactions.

The experimental results will be compared to the predictions of nuclear models which take into account explicitly the coupling between single-particle and collective states in order to explain the observed fragmentation and spreading of the single-particle strength.

\section{I - INTRODUCTION}

The topic of this paper, inner-hole and high-lying states in medium-heavy nuclei, is an example of a simple mode of excitation of the nucleus. In recent years, other modes such as giant resonances, spin-flip excitations, high-spin stretched states have been extensively studied and present a number of commun features with the present topic.

For example we are using transfer reactions to investigate high-lying single-particle strengths in nuclei. Such mode of excitation appears as "a giant resonance like structure" above a substantial background of more complicated states and therefore the 
problems of the extraction of the strength distribution, of the damping width, of the reaction model used in the data analysis, are very similar for such high-iying mode of excitations.

The questions I would like to address here are the following : how are modified the bare single-particle properties as one reaches higher and higher excitation energy in the nucleus ? What are the mechanisms responsible of the fragmentation and then the spreading of the single-particle strengths in nuclei?

Using the transfer reaction approach and some illustrative examples, our goal is to demonstrate that our present knowledge on single-particle strength distributions has been greatly enhanced during the past few years. The experimental results span a large domain of the nuclear. mass chart for both proton and neutron particle (hole) states. On the other hand the theoretical nuclear models have pointed out the key role played by the coupling between single particle motion and surface vibrations in order to reproduce the experimental resuits.

In the first part on this paper, I shall discuss briefly the recent results obtained in the study on one and two nucleon pick-up reactions. In the second part the new evidence for high-Tying particle strengths will be presented. A preliminary analysis of the experimental data in the framework of the distorted-wave Born approximation theory of nuclear reactions is carried out in order to extract the main characteristics (centroid energy, width, strength distribution) of such transitions. In the case of proton and neutron stripping reactions, a theoretical approach of the underlying background will be attempted, assuming that the main mechanism producing the observed background is due to the break-up of the projectile.

Finally the results obtained on high-spin outer subshells in ${ }^{116} \mathrm{Sn},{ }^{144} \mathrm{Sm},{ }^{208} \mathrm{~Pb}$ will be compared to the theoretical predictions of the quasi particle phonon and single particle vibrations nuclear models.

\section{II - ONE AND TWO NUCLEON PICK-UP FROM INNER SHELLS}

Perhaps one of the first example of observation of deep-hole states using a pick-up reaction, is the work reported by Sakai and Kubo on the ${ }^{118} \mathrm{Sn}\left({ }^{3} \mathrm{He}, \alpha\right)^{117} \mathrm{Sn}$ reaction 11. The residual energy spectra is shown in fig. 1 and a clear enhancement of the cross section is observed around $5.2 \mathrm{MeV}$ excitation energy in ${ }^{117} \mathrm{Sn}$. According to the authors, this excitation was attributed to the pick-up of a neutron from the next lower shell, e.g. $\lg _{9 / 2}, 2 p$ subshells as shown in fig. 1 .

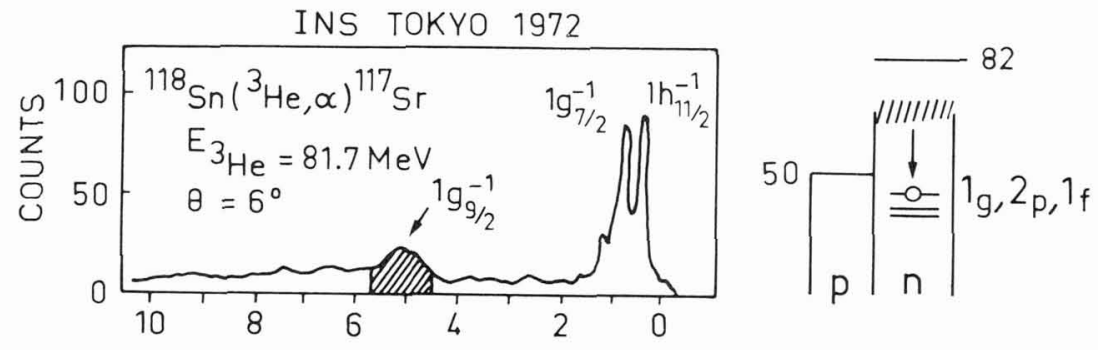

Fig.1 - (left) Residual energy spectra from the ${ }^{118} \mathrm{Sn}\left({ }^{3} \mathrm{He}, \alpha\right)^{117} \mathrm{Sn}$ reaction at $81.7 \mathrm{MeV}$ An enhancement of cross-section (bump) is clearly seen around $5.2 \mathrm{MeV}$ in ${ }^{117} \mathrm{Sn}$. (Right) Schematic picture of the neutron pick-up process to inner-shell for the $1 \mathrm{~g}, 2 \mathrm{p}$, $1 \mathrm{f}$ subshells in ${ }^{218} \mathrm{Sn}$ :

Very rapidly a growing amount of experimental data has , been accumulated and highlying hole strengths have been observed in $(p, d),(d, t),\left(d,{ }^{3} \mathrm{He}\right)$ and $\left({ }^{3} \mathrm{He}, \alpha\right)$ reactions at various energies from mass number $A=90$ to $A=209 / 2,3 /$. In recent review 
articles, a detailed information has been reported on the subject $/ 4,6 /$. Therefore in the following lines only a few examples will be discussed to emphasize the more recent advances.

II .1 - Selectivity in angular momentum transfer in one neutron. pick-up reactions

For a inner hole state located well below the Fermi sea, the pure hole configuration ( $1 h$ ) will mix through the residual interaction with the dense $(1 p-2 h)$ states leading a spreading of the hole-strength. In medium-heavy nuclei this spreading mechanism yields to overlapping structures corresponding to closely spaced inner-shells in the residual energy spectra. It is therefore of crucial importance that the selectivity of the reaction process enhances the excitation of a particular subshell $n \ell j$ with respect to neighbouring subshells and to the underlying background.

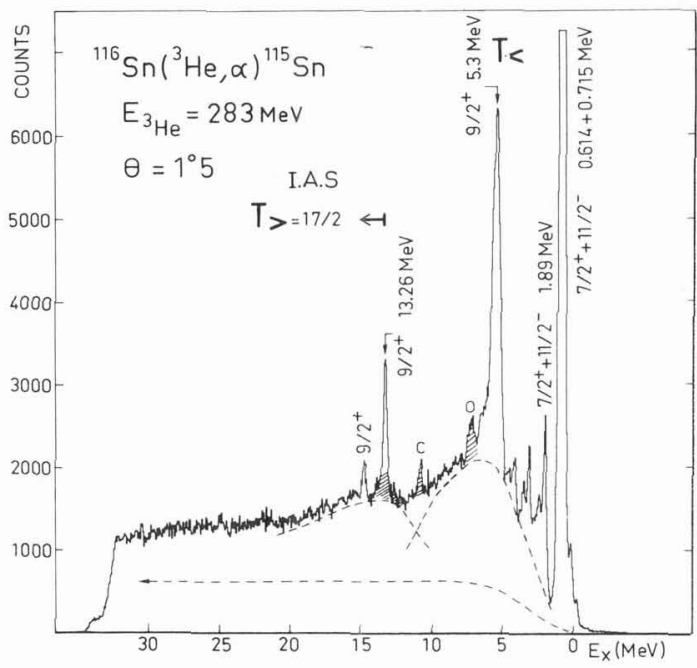

Fig.2 - Residual energy spectra from the ${ }^{116} \mathrm{Sn}\left({ }^{3} \mathrm{He}, \alpha\right)$ at $283 \mathrm{MeV}$ incident energy. The angular momentum matching conditions strongly select the $\ell=41 g_{9 / 2}$ inner hole strength mainly located in the peak around $5.3 \mathrm{MeV}$ in ${ }^{115} \mathrm{Sn}$.
This selectivity of the reaction process is clearly demonstrated in the spectrum of Fig. 2 where the $199 / 2$ inner-hole strength in ${ }^{115} \mathrm{Sn}$ is strongly excited in the $\left({ }^{3} \mathrm{He}, \alpha\right)$ reaction at $283 \mathrm{MeV} / 7 \%$. The large mismatching suppresses small $\ell$ transfer $(l=1,3)$ contributions in the whole range of excitation energy.

The well known concentration of $1 \mathrm{gg}_{\mathrm{g} / 2}$ strength at $5.3 \mathrm{MeV}$ dominates the spectra. In addition two new bumps are clearly identified at 7.5 and $15 \mathrm{MeV}$ respectively. The results of the analysis in the case of the $199 / 2$, $1 f_{5 / 2}$ and $1 f_{7 / 2}$ hole strengths in $1^{15} \mathrm{Sn}$ are summarized in Table I. A large fraction of the $1 f_{5 / 2}$ strength is 10cated between 8.6-11.6 MeV in reasonable agreement with the previous results of siemssen et a1. /8/. The main contribution to the higher energy bump $(11.5-18.5 \mathrm{MeV})$ is thus expected to be attributed to the $1 \mathrm{f} 7 / 2$ strength.
Table I

Fragmentation of the $189 / 2,1 \mathrm{f}_{5 / 2}$ and $1 \mathrm{f}_{7 / 2}$ neutron hole strengths in $115 \mathrm{Sn}^{\mathrm{a}}$ )

$\begin{array}{ccc}\begin{array}{c}\left\langle E_{x}\right\rangle \\ \mathrm{MeV}\end{array} & \mathrm{n} \ell j & \mathrm{C}^{2} \mathrm{~S} / 2 \mathrm{j}+ \\ 3.6-6.6 & 1 \mathrm{gg}_{9 / 2} & 0.56 \\ 6.6-8.6 & 1 \mathrm{gg}_{9 / 2} & 0.24 \\ 8.6-11.6 & 1 \mathrm{~g}_{9 / 2} & 0.14 \\ & 1 \mathrm{f}_{5 / 2} & 0.60 \\ 11.6-18.5 & 1 \mathrm{f}_{5 / 2} & 0.35 \\ & 1 \mathrm{f} 7 / 2 & \sim 1.00\end{array}$

Let us point out that the numbers quoted in Table I are strongly dependant on the assumed background level (dashed horizontal line in Fig.2). The investigation of a very large energy range (up to about $55 \mathrm{MeV}$ ) has been used in order to extrapolate the background line down to the lower energy region. 


\section{2 - The spin $J$ of the inner-hole state and transfer reaction with polarized beams}

In usual transfer reaction studies of deep-hole states, the $\ell$ value of the state has been determined by angular distribution measurements. The spin value $\mathrm{J}$ of the hole state, however, has been assigned only on the basis of shell model expectations and/or on sum-rule analysis. It is therefore highly desirable to measure the analyzing power $A y(\theta)$ of the transfered particle using a polarized incident beam since strong $J$ dependance of the analyzing power $A y(\theta)$ is expected from DWBA calculations.

Spin determinations of deeply-bound proton hole states have been reported by Wagner et $\mathrm{a} 7$. in the study of the $\left(\mathrm{d},{ }^{3} \mathrm{He}\right)$ reaction on a number of medium-heavy weigth target nuclei. $.5 \%$.

The first measurement of the spin $\mathrm{J}$ of the deep Ty-bound neutron hole states in the tin region was performed at Indiana University Cyclotron by the MSU-IndianaOrsay group using the ${ }^{120} \mathrm{Sn}(\overrightarrow{\mathrm{p}}, \mathrm{d})$ reaction at $90 \mathrm{MeV} / 9 \%$. In the mean time a study of the $(\vec{d}, t)$ reaction at $40 \mathrm{MeV}$ bombarding energy to inner-hole states in ${ }^{111,115} \mathrm{Sn}$ was also carried out using the ISN Grenoble cyclotron $/ 10 /$. To demonstrate the usefuiness of such studies, not only to determine the spin $J$ of the states but also to unravel the observed distributions in the case of overlapping subshells with different $n \ell j$ quantum numbers, I shall discuss in the following one example.

The $1 f_{5 / 2}$ and $1 f_{7 / 2}$ neutron-hole strengths in the $Z r$ region.

Deuteron spectrum from the ${ }^{9} \mathrm{Zr}(p, d)^{89} \mathrm{Zr}$ reaction is presented in Fig. 3 . Because of the angular momentum matching the ground state $(9 / 2)^{+}$and the $1.46 \mathrm{MeV}$ state $\left(5 / 2^{-}\right)$are very prominent among the low-lying levels. At excitation energies above $3.4 \mathrm{MeV}$, the gross structures, concluded to be mainly $1 \mathrm{f} 7 / 2$ hole states $13,11,12 /$, are clearly seen above a smooth background. The structures observed in the present experiment can be separated in two parts.

One of them is the bump with several peaks between $3.4 \mathrm{MeV}$ and $7.0 \mathrm{MeV}$ (labelled $\mathrm{A}$ in Fig. 3). Another one is the region lying between 7.0 and $21 \mathrm{MeV}(B, C, D)$. This latter region is structurless except for the sharp isobaric analog states (IAS) of low-lying levels of ${ }^{89} \mathrm{Y}$ ( labeled $T_{>}$).

The spin-parity of $7 / 2^{-}$proposed for all these structures $/ 3,11,12 /$ was based on the angular distributions and on spectroscopic factor arguments. But only analyzing power measurements could determine the $J$ value $\left(5 / 2^{-}\right.$or $\left.7 / 2^{-}\right)$since in a11 cases the angular distributions are characterized by an $\ell=3$ transfer $13,11,12 /$.

$\sigma(\theta)$ and $A y(\theta)$ for each area $A(3.4-$ 7.0 MeV $) B(7-11.6 \mathrm{MeV}), C(11.6-16 \mathrm{MeV})$ and $D(16-21 \mathrm{MeV})$ were obtained by integrating the yield above the background excluding the sharp peaks of the IAS around $9 \mathrm{MeV}$. The following arguments for $\mathrm{J}^{\pi}$ assignments are based on a comparison with the empirical analyzingpower, a method which has been successfully used in the case of the 199/2 hole state in ${ }^{119} \mathrm{Sn}$.

Fig.3 - Energy spectrum of the ${ }^{9} \mathrm{Zr}(\mathrm{p}, \mathrm{d}){ }^{89} \mathrm{Zr}$ reaction at $\mathrm{E}_{\mathrm{p}}=90 \mathrm{MeV}$. The major peak above $2.5 \mathrm{MeV}$ are labelled by excitation energies in MeV. The shaded and hatchedareas in the gross structure above $3.4 \mathrm{MeV}$ shows the $\left(1 \mathrm{f}_{7 / 2}\right)^{-1}$ and $\left(1 \mathrm{f}_{5 / 2}\right)^{-1}$ state distributions, $r \in$ spectively.

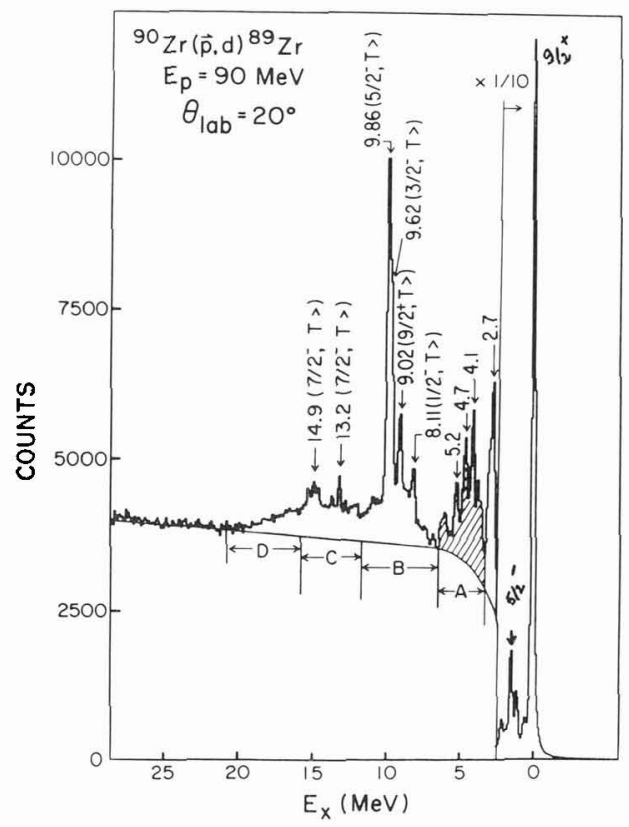


In Fig. 4 are shown $A y(\theta)$ for areas $A-C$ and the background under area $B$. The experimental $A y(\theta)$ clearly show that the main contribution to the areas $B$ and $C$ is from the same orbit and is different from $A$.

The main contribution to area $A$ is concluded to arise from the $1 f_{5 / 2}$ orbit by comparison with the Ay of the known $5 / 2^{-}$states at $1.46 \mathrm{MeV}$ in ${ }^{8}{ }^{9} \mathrm{Zr}$ and the $0.76 \mathrm{MeV}$ in ${ }^{57} \mathrm{Ni}$ as shown in the figure. Among the peaks in this region, the 4.1 and 5.2 MeV peaks have a non-negligible contribution of a $j=\ell+1 / 2\left(7 / 2^{-}\right)$transitions.

Areas $B$ and $C$ on the other hand are assigned to correspond mainly to $1 f_{7 / 2}$ neutron transfer since the Ay for these areas matches that for the known $2.57 \mathrm{MeV}, 7 / 2^{-}$ state in ${ }^{57} \mathrm{Ni}$ as shown in Fig.4.

In this case the analyzing power measurements establishes clearly the strong fragmentation of the $1 f_{5 / 2}$ hole strength up to $7 \mathrm{MeV}$ excitation energy in ${ }^{89} \mathrm{Zr}$ and help to locate the inner $1 f_{7 / 2}$ hole

state in the region $B$ and $C$. The overlapping regions between the $1 f_{5 / 2}$ and $1 f_{7 / 2}$ hole strengths can be determined through the study of neutron pick up reactions with a polarized beam.

The spin-orbit splitting between the $1 f_{5 / 2}$ and $1 f_{7 / 2}$ neutron subshells is of about $7 \mathrm{MeV}$ in reasonable agreement with the experimental value for proton subshe11s (5.10 Ref.5).

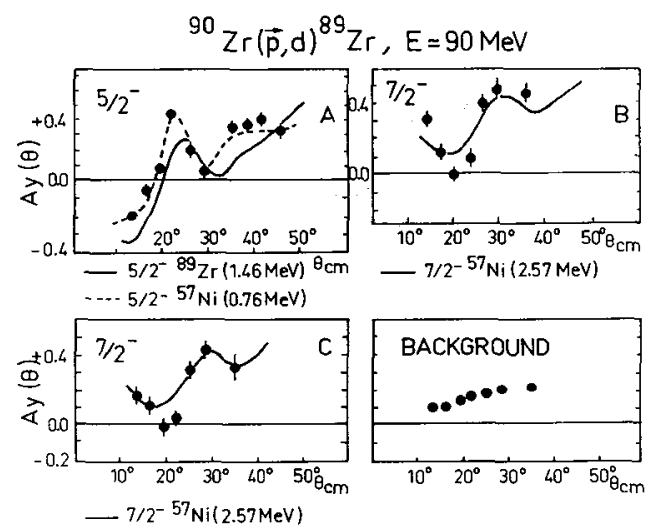

Fig.4 - Analyzing power for areas A,B,C and of the background. Solid lines are empirical distributions taken from ${ }^{58} \mathrm{Ni}(\overrightarrow{\mathrm{p}}, \mathrm{d}){ }^{57} \mathrm{Ni}\left(0.76 \mathrm{MeV}, 5 / 2^{-}\right)$ for area $\mathrm{A}$ and ${ }^{58} \mathrm{Ni}(\mathrm{p}, \mathrm{d}){ }^{57} \mathrm{Ni}\left(2.57 \mathrm{MeV} 7 / 2^{-}\right)$for areas $B$ and $C$. The dashed line is the empirical analyzing power for $5 / 2^{-}$from ${ }^{90} \mathrm{Zr}(\overrightarrow{\mathrm{p}}, \mathrm{d})^{89} \mathrm{Zr}$ $\left(1.46 \mathrm{MeV}, 5 / 2^{-}\right)$state.

\section{3 - Two nucleon strength distributions from $(p, t)$ and $\left(\alpha,{ }^{6} \mathrm{He}\right)$ reactions}

Another type of simple excitation mode of the nucleus, is the high-lying two-neutron hole states which may be related to the general phenomena of deep-hole states, observed in one nucleon pick-up reactions. Most of the existing data on two-nucleon strength distributions comes from $(p, t)$ reactions carried out at rather low incident energy $(<60 \mathrm{MeV})$, with special emphasis on low-lying, low spin configurations.

The first evidence for high-iying two neutron hole states was reported by Crawley et al $/ 13 /$ in their. ( $p, t)$ studies at $42 \mathrm{MeV}$ on tin isotopes. A systematic investigation of this phenomena was undertaken on $\mathrm{Cd}$, Te, Sm isotopes at $42 \mathrm{MeV}$ and on tin isotopes at $90 \mathrm{MeV} / 14 /$. Detailed information can be found in Refs,4,6. As a example the $118,120 \mathrm{Sn}(p, t)^{116},{ }^{118} \mathrm{Sn}$ energy spectra are shown in Fig.5. The bump observed in $(p, t)$ reactions was first interpreted in terms of deep-lying pairing vibrations or removal of two nucleons from inner shells $(1 \mathrm{~g} g / 2,2 p)$. However the extracted angular distributions at 42 as we 11 as at $90 \mathrm{MeV}$ incident energy were not reproduced by a unique $L$ transfer but rather by the total of al1 transitions arising from particles picked up from inner shells $\left(1 g_{9 / 2}, 2 p\right)$ and/or from valence + inner shel1s (e.g. $d_{5 / 2}+g_{9 / 2}$ etc ...). Soon after 1 t was pointed out by Nomura $/ 15 /$ that the bump arises simply from two holes : the deep-Tying $(d)$ and the valence $(v)$. 
Additional evidence for such a conclusion was obtained in the study of the ( $p, d)$ reaction on two odd tin isotopes ${ }^{117,119} \mathrm{Sn}$ at $42 \mathrm{MeV} / 16 /$. The resulting spectra from $(p, d)$ and $(p, t)$ reactions leading to the same final nucleus are shown in Fig.5. A broad structure at about the same excitation energy is observed in the two reactions.

In $(p, d)$ reactions, these broad peaks can only come from the coupling of one hole in the inner-shell $(199 / 2,2 p)$ with the hole near the Fermi surface $\left(d_{5 / 2}, g_{7 / 2}\right)$ which is present in the $117,119 \mathrm{Sn}$ ground state $(v+d$ configurations). In $(p, t)$ reactions both $v+d$ and $2 d$ ( 2 deep holes) could be excited. The comparison of spectra of the Fig. 5 suggests strongly that the bumps populated in $(p, t)$ studies are mostly due to the $v+d$ configurations $/ 16 /$.
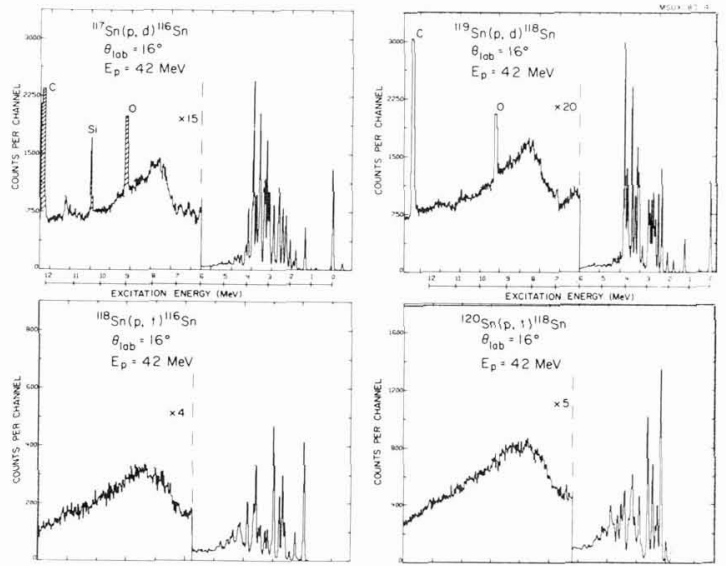

Alternatively, the $\left(\alpha,{ }^{6} \mathrm{He}\right)$ appears as a new and attractive tool to explore the two neutron-hole strengths, due to its expected selectivity towards large $L$ angular momentum transfer at high incident energy. The high incident energy of the $\alpha$ particle (218 MeV) and proton (168 MeV) beams of the Orsay synchrocyclotron are well suited to a comparative study of the $\left(\alpha,{ }^{6} \mathrm{He}\right)$ and $(p, t)$ reactions on mediumheavy targets $\left({ }^{58} \mathrm{Ni},{ }^{90} \mathrm{Zr},{ }^{126} \mathrm{Sn},{ }^{144} \mathrm{Sm}\right.$ and $\left.{ }^{208} \mathrm{~Pb}\right)$.

Angular distributions were measured with the high-resolution spectrometer from $2^{\circ}$ to $18^{\circ}$. Clean identification of the outgoing tritons $\left({ }^{6} \mathrm{He}\right)$ was achieved. The overa11 energy resolution was $220 \mathrm{KeV}$ for $\left(\alpha,{ }^{6} \mathrm{He}\right)$ and $130 \mathrm{KeV}$ for $(p, t)$ reactions.

Typical spectra obtained on ${ }^{116} \mathrm{Sn}$ target are displayed in Fig.6. The ${ }^{114} \mathrm{Sn}$ spectrum is dominated by two structures around $3.4 \mathrm{MeV}$ and $8.0 \mathrm{MeV}$ respectively. The broad structure around $8 \mathrm{MeV}$ confirms the previous results from $(p, t)$ studies. However for the first time we can point out two components, differently excited in the two reactions (see Fig.6)./18/

The first component, centered at $7.4 \mathrm{MeV}$ is preferentially excited in the $(p, t)$ reaction. The bump as a whole have a width (FWHM) of $2.0 \pm 0.2 \mathrm{MeV}$. A préliminary analys is of the $\left(\alpha,{ }^{6} \mathrm{He}\right)$ reaction shows that the observed cross-section is mainly due to the 1 deep-1 valence hole $\left[\left(1 g_{g / 2}\right)^{-1}\left(2 d_{5 / 2}, 2 d_{3 / 2}\right)^{-1}\right]_{6^{+}}$configurations. Similarly the large enhancement at $3.4 \mathrm{MeV}$ is also dominated by $6^{+}$states arising mainly from valencevalence $\left[(1 \mathrm{~g} 7 / 2)^{-1} \otimes\left(2 \mathrm{~d}_{5 / 2}\right)^{-1}\right]$ confi-

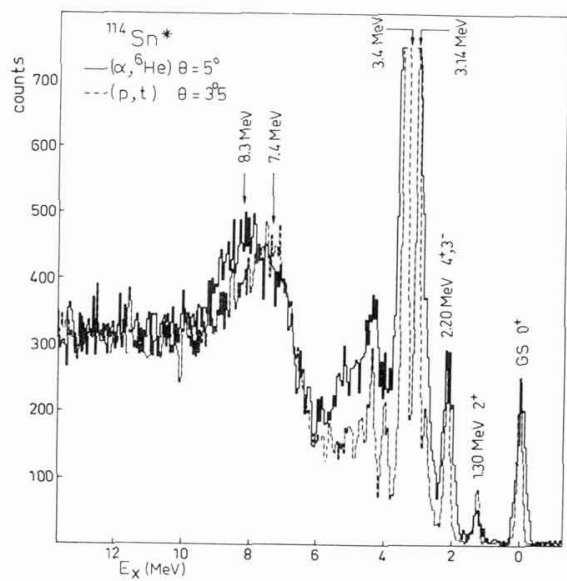

Fig.6 - Triton and ${ }^{6}$ He energy spectra from the $(p, t)$ and $\left(\alpha,{ }^{6} \mathrm{He}\right)$ reactions on ${ }^{116} \mathrm{Sn}$ target. gurations. 
Theoretical investigations of two quasiparticle strengths in the $\mathrm{Sn}$ isotopes by Soloviev et $21 . / 17 /$ are in very good agreement with our conclusions.

Finally the two-deep (2d) hole strengths should be around $11.5-13.5 \mathrm{MeV}$ in ${ }^{114} \mathrm{Sn}$. We did not find any significant enhancement of the $(p, t)$ or $\left(\alpha,{ }^{6} \mathrm{He}\right)$ cross-sections up to $17 \mathrm{MeV}$ excitation energy in ${ }^{114} \mathrm{Sn}$.

The second subject of interest related to the study of the $\left(\alpha,{ }^{6} \mathrm{He}\right)$ reaction is the understanding of the reaction mechanism since the existing data are very scarce. Therefore, the ${ }^{208} \mathrm{~Pb}\left(\alpha,{ }^{6} \mathrm{He}\right)^{206} \mathrm{~Pb}$ reaction $/ 18 /$ was chosen due to the rather pure and well spaced two-neutron hole components up to $4.2 \mathrm{MeV}$ excitation energy in ${ }^{206} \mathrm{~Pb}$. Typical energy spectrum from $(\alpha, 6 \mathrm{He})$ reaction on ${ }^{208} \mathrm{~Pb}$ is displayed in Fig. 7.

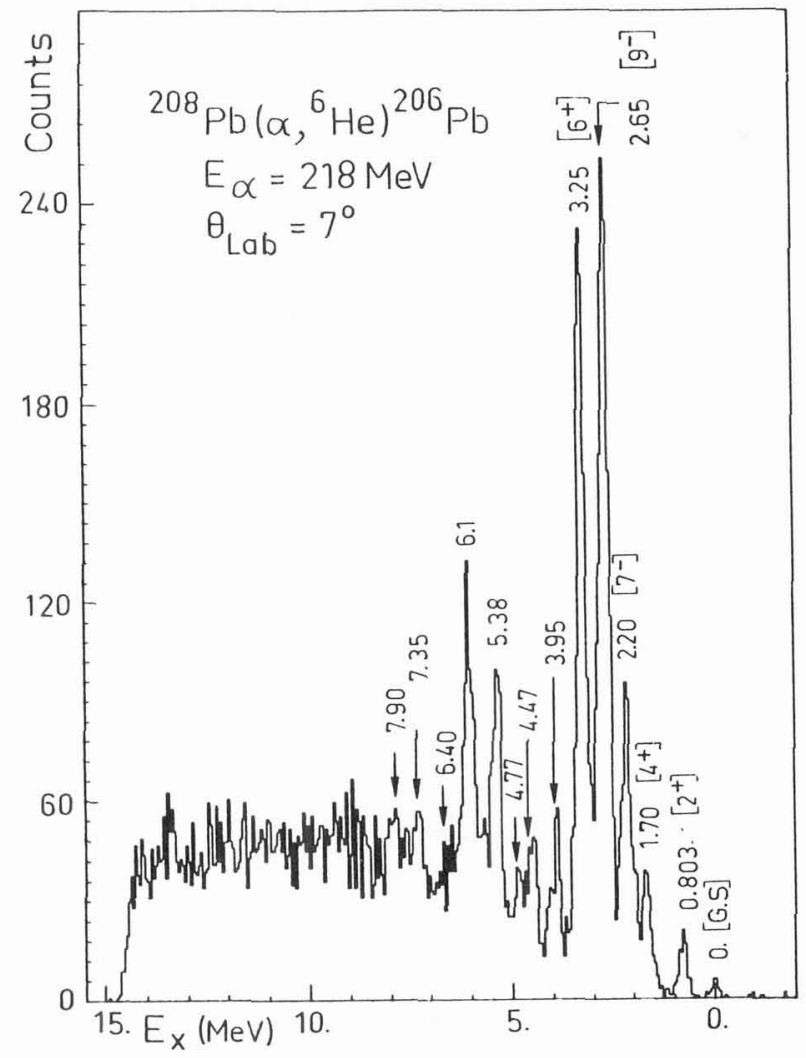

Fig.7 $-{ }^{6} \mathrm{He}$ energy spectrum from the ${ }^{208} \mathrm{~Pb}\left(\alpha,{ }^{6} \mathrm{He}\right)$ reaction recorded at $\theta=7^{\circ}$ lab angle.

The selectivity of this reaction at $218 \mathrm{MeV}$ is clearly demonstrated. In ${ }^{206} \mathrm{~Pb}$ the first low-lying, low-spin states $0^{+}, 2^{+}, 4$ are weakly populated as compared to the $\mathrm{Ex}=2.65 \mathrm{MeV}, \mathrm{J}^{\pi}=9^{-}$and the $\mathrm{Ex}=3.25 \mathrm{MeV}, \mathrm{J}^{\pi}=6^{+}$states.

Experimental angular distributions are presented in Fig.8. These experimental angular distributions appear to have a characteristic and well structured $L$ dependence, a feature which was not expected for transfer reactions athigh incident energy.

Considering the fairly well matched states, we wish to point out that the present experiment, in agreement with preliminary DWBA calculations support the following rule. 
The reaction strongly enhances natural parity levels with spin and configuration

$$
J^{\pi}=\left|\left(j_{1}=\ell_{1}-1 / 2\right)^{-1}\left(j_{2}=l_{2}+1 / 2\right)^{-1}\right|_{\text {Jmax }}
$$

An additional selectivity is observed if $\ell_{1}=\ell_{2}$. This is the case for the $6^{+}$

level at $3.25 \mathrm{MeV}$, the main component is $J^{\pi}=!\left(f_{5 / 2}\right)^{-1}\left(f_{7 / 2}\right)^{-1} / 6^{+}$.

On that basis one may assume that the new level at $6.1 \mathrm{MeV}$ is the $\mathrm{J}_{\max }^{\pi}=8^{+}$ member of the $\left|\left(h_{9 / 2}\right)^{-1}\left(f_{7 / 2}\right)^{-1}\right|_{8^{+}}$ multiplet predicted around $6.5 \mathrm{MeV} / 19 /$.

No indication of any enhancement of crosssection arising from the population of 1 deep $\left(1 h_{11 / 2}\right)+1$ valence $\left(1 f_{5 / 2}, 1 h_{9 / 2}\right)$ hole state has been found up to $15 \mathrm{MeV}$ excitation energy.

Summarizing this new approach we would like to stress that the $\left(\alpha,{ }^{6} \mathrm{He}\right)$ reaction at $218 \mathrm{MeV}$ could be used as a new and attractive spectroscopic tool in order to study both low and high-lying two neutron-hole pairs coupled to the maximum spin value in medium-heavy nuclei.

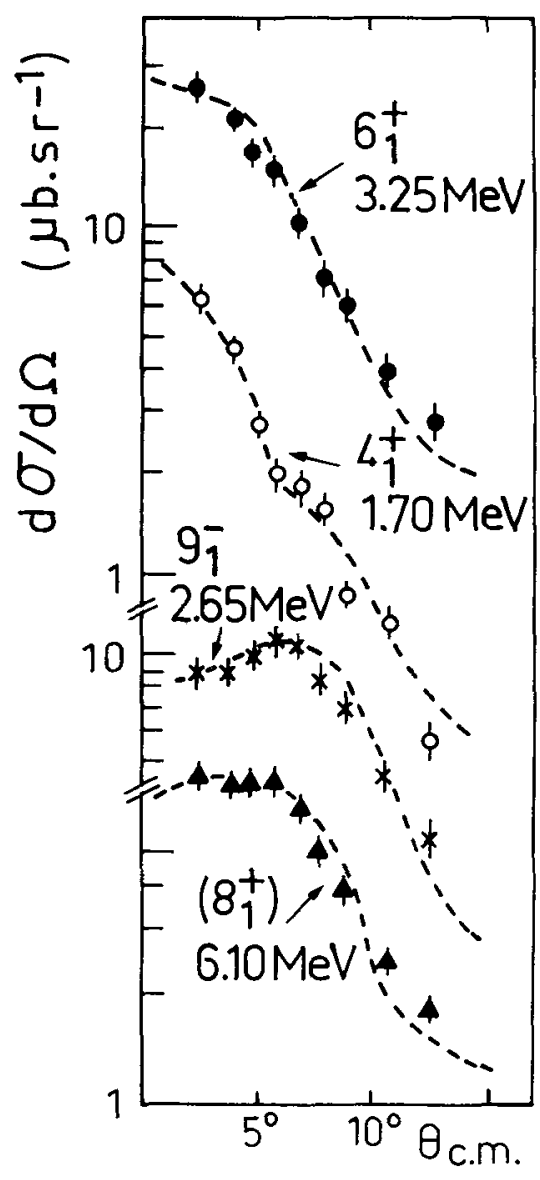

Fig. 8 - Typical angular distributions from the ${ }^{20} \mathrm{~Pb}\left(\alpha,{ }^{6} \mathrm{He}\right)$ reaction at $218 \mathrm{meV}$ Dashed lines are only indicated to guide the eye.

\section{II - HIGH-LYING SINGLE - PARTICLE STRENGTHS VIA STRIPPING REACTIONS}

With the exception of the well-known studies of proton stripping to quasi-bound or unbound Isobaric Analog States (IAS) $/ 20 /$, pratically no information is available on high-lying "particle" states. The properties of the $T_{>}$part of the singleparticle strengths for subshells located well above the Fermi sea are rather well established. On the other hand the $T_{<}$part, where the bulk of the single-particle strength is concentrated, is poorly known. Therefore, one nucleon stripping reactions appear as a powerful tool in order to reach the properties of the high-lying proton and neutron states in a way similar to the one employed for deeply-bound states. 
A new important step has been recently made towards the use of the one nucleon transfer reactions for the study of high-lying nuclear excitations. A new type of resonance $1 \mathrm{ike}$ structure has been observed in the study of proton stripping reactions $(\alpha, t)$ and $\left({ }^{3} \mathrm{He}, d\right)$ on a number of medium-heavy target nuclei $\left({ }^{90} \mathrm{Zr},{ }^{116} \mathrm{Sn}\right.$, $\left.1{ }^{44} \mathrm{Sm},{ }^{208} \mathrm{~Pb}\right) / 21 /$.

The general behaviour of the particle response function has been recently extended to the neutron case with the help of the $\left(\alpha,{ }^{3} \mathrm{He}\right)$ reactions. In the following, the empirical systematics will be presented and the results from the data analysis compared to the predictions of nuclear models. The close connection between high-lying and deeply-bound states will be emphasized.

\section{III.1 - The $(\alpha, t)$ and $\left({ }^{3} \mathrm{He}, \mathrm{d}\right)$ reaction to high-lying proton states in ${ }^{145} \mathrm{Eu}$}

The first experimental evidence of high-lying proton excitations in heavy nuclei was obtained while studying the ${ }^{144} \mathrm{Sm}(\alpha, t)$ at $80 \mathrm{MeV}$, complemented by an investigation of the ( $\left.{ }^{3} \mathrm{He}, \mathrm{d}\right)$ process at $240 \mathrm{MeV}$.

The $(\alpha, t)$ experiment was performed with $\alpha$-particlebeams delivered by the Grenoble cyclotron. Outgoing tritons were detected by a gaz delay-line counter backed by two plastic scintillators in the focal plane of the QD spectrometer. Both high $(50 \mathrm{keV})$ and low (230 KeV) energy resolution spectra were recorded using thin $\left(0.5 \mathrm{mg} / \mathrm{cm}^{2}\right)$ and thick $\left(6 \mathrm{mg} / \mathrm{cm}^{2}\right)$ target up to $22 \mathrm{MeV}$ excitation energy.

Angular distributions were measured from $1^{\circ} 75$ to $18^{\circ}$ (laboratory angle) in $3^{\circ}$ step. The ${ }^{145} \mathrm{Eu}$ energy spectrum from the $(\alpha, t)$ reaction is presented in Fig. 9a. At low excitation energy, one observed a selective population of the $1 \mathrm{~h} 11 / 2$ state due to the known selectivity of this reaction for large $\ell$ values (here $\ell=5,6$ ). Before this study no information was available for proton states above $2.5 \mathrm{MeV}$.

At high excitation energy ( $E x>4 \mathrm{MeV}$ ), two broad bumps are strongly excited above a substantial background. Their spacing in energy, their respective width, and in particular the fact that the second bump (B) appears as a shoulder of the main structure $(A)$ recall the similar features observed in the study of deeply bound $1 g_{9} / 2$ and $2 p$ hole states in $t$ in isotopes $/ 2-4,6-8,10-12 /$. Around $14 \mathrm{MeV}$ a sharp peak corresponding to the $1 h_{11 / 2}$ and $1_{i} / 3 / 2$ IAS is observed for the first time.

In order to gain further insigth in the study of this phenomena, the same range of excitation energy in ${ }^{145} \mathrm{Eu}$ was investigated by means of the reaction ${ }^{144} \mathrm{Sm}\left({ }^{3} \mathrm{He}, d\right)^{145} \mathrm{Eu}$ at $240 \mathrm{MeV}$ using the ${ }^{3} \mathrm{He}$ beam from the Orsay synchrocyclotron and a large magnetic spectrometer. The emerging deuterons were detected by two multiwire proportional chambers backed by two plastic scintillators. The resulting energy spectrum is displayed in Fig. 9b. The broad structures $A$ and $B$ are also observed in the presented spectrum, their positions and widths being consistent with the $(\alpha, t)$ results. Their relative yields in the two spectra are quite different due to the different selectivity in momentum transfer of the two processes.

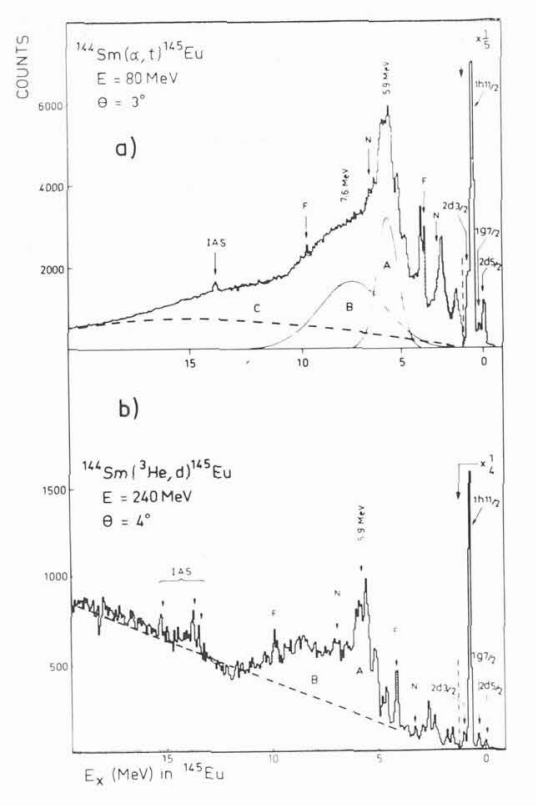

Fig.9a,b - Triton (deuteron) energy spectra from the reactions ${ }^{144} \mathrm{Sm}(\alpha, t)$ (upper part) and $1{ }^{44} \mathrm{Sm}\left({ }^{3} \mathrm{He}, d\right)$. The dashed line shows the background line shape from the PWBU model. The solid curve show the result of the fitting procedure (Background C, Gaussian peaks $A$ and $B$ ). 
One plausible explanation for these peaks is that they arise from proton states in the next major shell consisting of closely spaced high-spin orbitals, e.g. $2 f_{7 / 2}$, $1 \mathrm{hg} / 2$ and $1 \mathrm{i} 13 / 2$. In other words we are investigating "outer subshells".

In order to test this assumption a detailed analysis of the data has been carried out.

\section{III.1.1 - The background line shape and break up of the projectile}

The extraction of the centroïd energies, widths and differential cross-sections of the "peaks" $A$ and $B$ implies an assumption on the background line shape. Since the spectral shapes of $\alpha$ and ${ }^{3} \mathrm{He}$ particle break-up processes at incident energy ranging from 20 to $40 \mathrm{MeV} /$ nucleon are well explained by a simple plane wave break -up model $122,23 /$, (PWBU), similar calculations including corrections due to the Coulomb force were carried out in order to have a consistent description of the background. The theoretical predictions were normalized to the data at forward angles $\left(\theta<6^{\circ}\right)$ and at high excitation energy $\left(E_{X}>20 \mathrm{MeV}\right)$. The results are shown as dashed lines in Figs $9 \mathrm{a}$ and $9 \mathrm{~b}$.

This simple model was not able to reproduce the line shape of the continuum crosssection in the case of the $(\alpha, t)$ reaction at $80 \mathrm{MeV}$ on heavy nuclei, whereas for the $\left({ }^{3} \mathrm{He}, \mathrm{d}\right)$ reaction the predictions are in good agreement with the experimental data.

\section{III.1.2 - Data_reduction and DWBA analysis}

The failure of this simple PWBU model in the case of the $(\alpha, t)$ reaction lead us to use the so-called "empirical background Tine shape". In Fig. 9a the solid 1ine, which smoothly connects the structureless part of the spectrum to the minima of cross sections near $3.5 \mathrm{MeV}$, represents that assumption (region C). The remaining part of the cross-section was fitted by two Gaussian peaks having different widths. The results of this fitting procedure is shown in Fig. 9 a (Gaussian peaks $A$ and $B$ ).

The parameters used in the DWBA analysis were successfully tested on known low lying states $/ 21 /$. Moreover, since the observed gross structures are located well above the proton threshold, unbound form factors using Gamow functions /20/ were

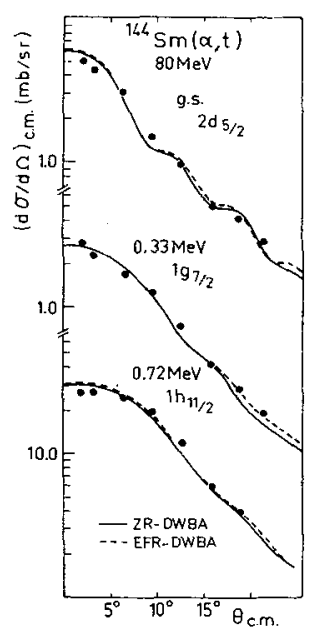

Fig. 10 - Experimental data and theoretical DWBA curves from the ${ }_{144} \operatorname{Sm}(\alpha, t){ }^{145} \mathrm{Eu}$ reaction to low lying states.

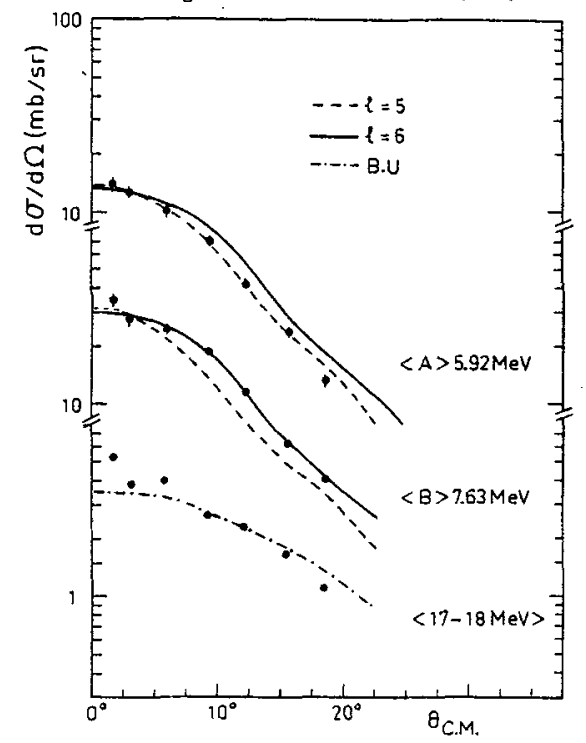

Fig.11 - Angular distributions from the reaction ${ }^{14} \mathrm{Sm}(\alpha, t)$ to high-lying proton states. Solid and dashed lines are DWBA calculations for the indicated lvalues. Centroīd energies of the bumps are given. The dot-dashed curve correspond to the prediction of the PWBU model. 
employed in the analysis. Typical experimental angular distributfons and theoretical DWBA curves for both well-known low-lying states $\left(2 \mathrm{~d}_{5 / 2}, 1 \mathrm{~g}_{7 / 2}\right.$ and $1 \mathrm{~h}_{11 / 2}$ levels in $145 \mathrm{Eu}$ ) and the high-lying "peaks" $A$ and $B$ are shown in Figs. 10 and 11 , respectively.

A very good agreement is found between the data and DWBA calculations if one assumes an $\ell=5$ transfer for the bump $A$ and an $\ell=6$ transfer for the bump. B. For completeness one should mention that an $\ell=3$ transfer does not reproduce the trend of the experimental data but a $20 \%$ mixing of $\ell=3$ in the bump $A$ will not change significantly the quality of the fit. As regard to the deduced strengths, the results. of the DWBA analysis indicates that the full $\&=51 \mathrm{~h} / 2$ proton strength is found in the bump $A$ with a possible $20 \%$ admixture of $\ell=3,2 f_{7 / 2}$ strength and that the $\ell=61 i 13 / 2$ proton strength is concentrated in region $B$. "Finally the angular distributions obtained for the background (see Fig. 11) agree reasonably well with the prediction of the PWBU model.

\section{III.2 - Empirical systematics of high-lying proton states}

Under the same experimental conditions the $(\alpha, t)$ reaction at $80 \mathrm{MeV}$ has been studied on ${ }_{208} \mathrm{~Pb}$ and ${ }^{116} \mathrm{Sn}$ target. The residual energy spectra of the three final nuclei ${ }^{117} \mathrm{Sb}$, ${ }^{4} \mathrm{Eu}$ and ${ }^{208} \mathrm{Bi}$ are displayed in Fig. 12 . We would like to point out the similarity of the empirical background line shapes which reinforces our assumption, namely the main part of the cross-section found in the underlying continuum comes from the break-up of the $\alpha$-particle. Weak dependance of the background yield versus the atomic number $A$ is one of the characteristics of the break-up process. Of particular importance is the calculation of the continuum in the case of the ${ }^{208} \mathrm{~Pb}(\alpha, t)$ reaction by $\mathrm{R}$. Shyam et $\mathrm{a}$. The double differential cross-section $\mathrm{d}^{2} \sigma / \mathrm{d}_{\Omega} \mathrm{dE}$ is calculated in the framework of the DWBA approach for projectile break-up and takes into account elastic and inelastic reactions of the break-up fragments. The deduced curve is plotted in absolute value with no additiona 7 normalization. The shape of the underlying continuum is well reproduced by the reaction model whereas the absolute values are off by 40 to $50 \%$. Both optical mode 1 parameters and zero-range approximation may account easily for the missing cross-sections.

Turning now to the high-lying strength distributions the analysis of the bump $A$ in ${ }_{117} \mathrm{Sb}$ shows that most of the proton strength located in the outer high-spin subshelTs, e.g. $1 h_{9 / 2}$ and $1 i_{13 / 2}(Z>82)$ is found in that energy region. The bump is located around $10.8 \mathrm{MeV}$ with a width of $5.5 \mathrm{MeV}$.

In the case of ${ }^{209} \mathrm{Bi}$ a very detailed analysis has been carried out. The results can be summarized as follows:

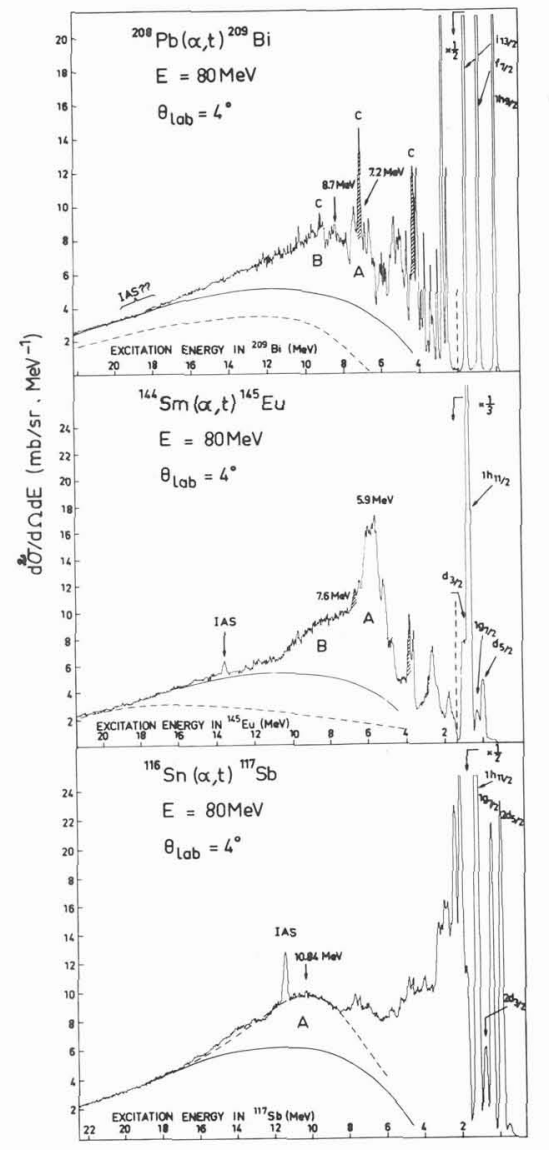

Fig.12 -Residual energy spectra from the $(\alpha, t)$ reaction on ${ }^{116} \mathrm{Sn}, 144 \mathrm{Sm}$ and ${ }^{208} \mathrm{~Pb}$ targets. The solid lines are the assumed background. The dashed curves are the PWBU model predictions. The dashed curve in the case of $2{ }^{09} \mathrm{Bi}$ is the DWBA break-up calculations (abso1ute values, see text). 
In addition to the wel1-know low-lying and rather pure single particle states $\left(1 h_{9 / 2}, 2 f_{7 / 2}, 1 i_{13 / 2}\right)$ a number of $\ell=6$ transitions are found between 2 to $6 \mathrm{MeV}$ excitation energy in ${ }^{209} \mathrm{Bi}$.

They may correspond to the fragmentation of the $1 i_{13 / 2}$ proton strength. However significant fragments of the $\ell=7 \quad i_{15 / 2}$ strength are also found in that energy range.

In the region labelled $A$ in Fig. 12 we found a small fraction of the $l=6 \quad 1 i_{11 / 2}$ and $\ell=42 \mathrm{~g}_{9} / 2$ proton. In region $B$ most of the single particle strength from the high-spin outer subshelis $1 i_{11 / 2}$ and $1 j_{15 / 2}$ is concentrated.

The above mentioned conclusions are confirmed by the ( $\left.{ }^{3} \mathrm{He}, \mathrm{d}\right)$ study of the same target nuclei as it is shown in Fig. 13.

The structures observed in the $(\alpha, t)$ study are clearly confirmed by the $\left({ }^{3} \mathrm{He}, \mathrm{d}\right)$ experiments. Again it is worth pointing out that the PWBU model reproduces fairly well the slope and shape of the underlying continuum for all the investigated target nuclei. The characteristics of the high-lying proton states in ${ }^{117} \mathrm{Sb}$ ${ }_{145} \mathrm{Eu}$ and ${ }^{209} \mathrm{Bi}$, centroĩd energy spreading, width and spectroscopic strength are presented in Table II.

These quantities being deduced from the data reduction and the analysis, we can compare now our results to the predictions of nuclear models.
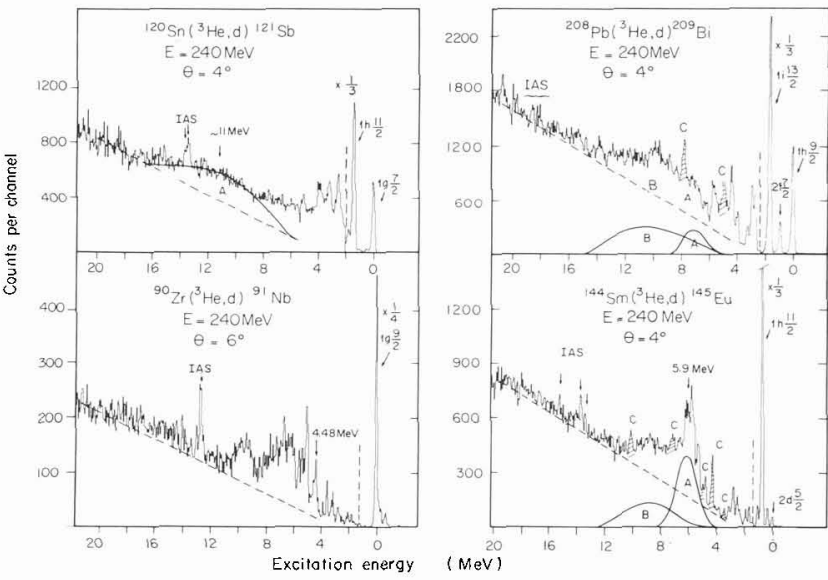

Fig. 13 - Residual energy spectra from the ( ${ }^{3} \mathrm{He}, \mathrm{d}$ ) reaction at $240 \mathrm{MeV}$ on ${ }^{0} \mathrm{Zr},{ }^{120} \mathrm{Sn},{ }^{144} \mathrm{Sm}$ and ${ }^{208} \mathrm{~Pb}$ target nuclei. The dashed line is the prediction of the PWBU model for the underlying background.

Table II - Summary of the results from the analysis of the $(\alpha, t)$ and $\left({ }^{3} \mathrm{He}, \mathrm{d}\right)$ reactions to high-1ying proton states in ${ }^{117} \mathrm{Sb},{ }^{145} \mathrm{Eu}$ and ${ }^{209} \mathrm{Bi}$.

\begin{tabular}{|c|c|c|c|c|c|}
\hline \multicolumn{2}{|c|}{ Nucleus/Bump } & $\mathrm{E}(\mathrm{MeV})$ & $\Gamma(\mathrm{MeV})$ & $n \& j$ & $\mathrm{C}^{2} \mathrm{~S}$ \\
\hline${ }^{117} \mathrm{Sb}$ & A & $10.84 \pm 0.2$ & $5.5 \pm 0.5$ & $1 i_{13 / 2}+1 h_{9 / 2}$ & $\sim 0.9$ \\
\hline${ }^{145} \mathrm{Eu}$ & A & $5.92 \pm 0.20$ & $1.23 \pm 0.15$ & $1 \mathrm{~h}_{9 / 2}$ or & 0.97 \\
\hline & & $* 5.9 \pm 0.10$ & $0.9 \pm 0.10$ & $1 h_{9 / 2}+2 f_{7 / 2}$ & $0.75 ; 0.19$ \\
\hline & B & $7.63 \pm 0.40$ & $4.0 \pm 0.45$ & $1 i_{12}$ & 0.94 \\
\hline & & $* 8.60 \pm 0.50$ & $4.0 \pm 0.50$ & 1372 & \\
\hline${ }^{209} \mathrm{Bi}$ & A & $7.2 \pm 0.2$ & $0.63 \pm 0.20$ & $1 i_{11 / 2}$ & 0.15 \\
\hline & & $* 7.15 \pm 0.15$ & $1.50 \pm 0.30$ & $111 / 2$ & \\
\hline & B & $8.7 \pm 0.5$ & $5.3 \pm 1.0$ & $1 i_{11 / 2}+1 j_{15 / 2}$ & $\sim 1.0$ \\
\hline & & $* 10.3 \pm 0.5$ & $5.7 \pm 1.0$ & & \\
\hline
\end{tabular}

* From the $\left({ }^{3} \mathrm{He}, \mathrm{d}\right)$ spectra. 


\section{III.3 - Comparison with nuclear models}

During the recent years, the large amount of experimental data on inner-hole strengths in medium-heavy nuclei lead to a similar growth of theoretical papers on the fragmentation and damping of nuclear excitation $/ 25,28 /$.

Two theoretical approachs were rather successful in reproducing the empirical systematics of the inner-hole strength distributions.

The Dubna group /25/ using the quasi particle-phonon nuclear model has investigated the fragmentation mechanism. The necessity of taking into account the "quasiparticle plus two phonons" components and of calculating with a large phonon basis has been shown. On the other hand, a theoretical model that has found a considerable success is based on a damping mechanism in which the simple excitation mix with the surface vibrations. A self consistent mixing between single particle motion (calculated with the Hartree-Fock Hamiltonian), and vibrationsfrom RPA calculations coutb be achieved $/ 26,28 /$ and the resulting strength functions are compared to the empirical systematics. In the case of high-lying particles since no data was avajlable, no calculations were reported until now. Soon after the publication of our first results, predictions of proton strength functions became available.

In Fig. 14 are presented the theoretical strength functions (unit of strength per unit energy interval) for the high-lying $2 f_{7 / 2}$, ihg/2 and $1 i_{13 / 2}$ outer subshells in ${ }^{145} \mathrm{Eu}$ calculated within the framework of the quasi particle-phonon model $/ 29 /$.

The results of the calculation are compared to the experimental values (deduced from the analysis of $(\alpha, t)$ and $\left({ }^{3} \mathrm{He}, \mathrm{d}\right)$ reactions). In the figure is also indicated the level schenie of proton quasi-particle state calculated in a Woods Saxon potential. The theoretical strength. functions differ noticeably from each other. However the values of the second moment $\sigma$ of the threedistributions are close.

The agreement with experiment is quite good for the $i 13 / 2$ subshe 11 whereas the damping of the $2 \mathrm{f} 7 / 2$ and 1 h $/ 2$ strengths is rather large compared to the experimental ones. One may notice also a energy difference of about $1.0 \mathrm{MeV}$ between the centroid energies of the theoretical and experimental strength distributions. Such desagreement may be explained by the procedure used in the data analys is (Gaussian shapes) and/or by a less accurate determination of the experimental strengths in the overlapping regions.

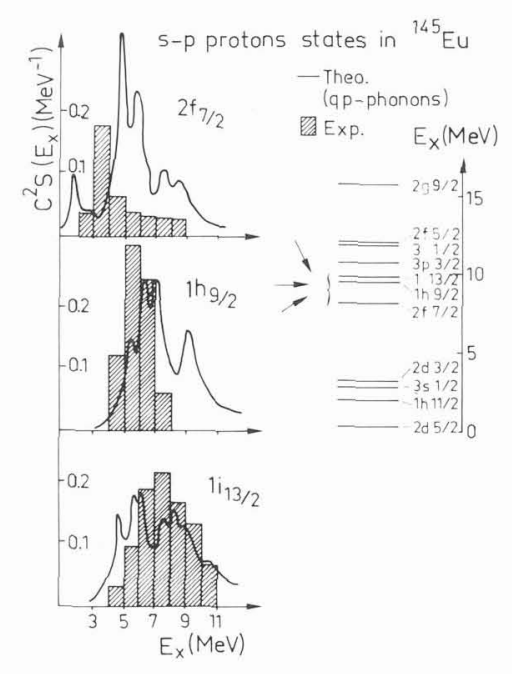

Fig. 14 - Comparison between theoretica1 /29/ and experimental proton strength distributions for high-lying subshells in ${ }^{145} \mathrm{Eu}$. In Fig. 15, similar comparison is made in the case of the outer $1 i_{11 / 2}$ and $1 j_{15 / 2}$
subshelis in ${ }^{209} \mathrm{Bi}$. Here again a qualitative agreement is achieved.

The theoretical calculations confirm the strong overlap between the $1 i_{11 / 2}$ and $1 j_{15 / 2}$ strength functions in ${ }^{209} \mathrm{Bi}$. The local maxima near. $7.2 \mathrm{MeV}$ may correspond the region. labelled $A$ in the corresponding ${ }_{209} \mathrm{Bi}$ spectrum (see Fig. 12). For the same nuclei, e.g. ${ }^{209} \mathrm{Bi}$, calculations in the framework of the single-particle vibrations coupling nuclear model has been carried out by N.Van Giai et a1./30/. 


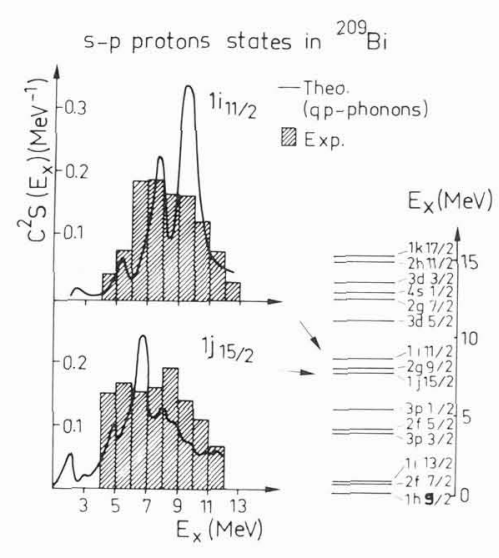

Fig. 15 same as Fig.14 for the case of the $1 i_{11 / 2}$ and $1 j_{15 / 2}$ proton strengths in $209 \mathrm{Bi}$.

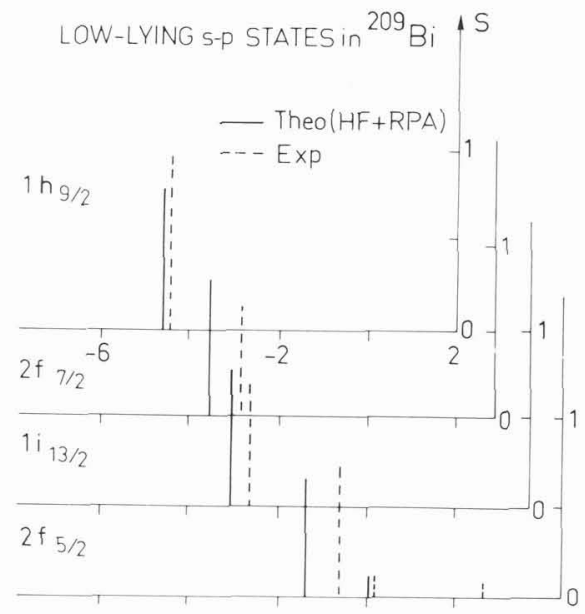

Fig. 16 - Comparison between theoretical/30/ and experimental strengths for the low-lying single particle states in ${ }^{209} \mathrm{Bi}$.

The theoretical predictions are made for both the low-7ying and high-lying proton subshel1s, giving an additional interest to such approach since the whole single particle proton spectrum can be compared to the experimental results.

In Fig. 16 the theoretical predictions for the strength distributions of the low-lying $1 \mathrm{hg} / 2,2 f_{7 / 2}$ and $1 i_{13 / 2}$ subshells are compared to the experimental results. It is worth pointing out that there is no free parameter in such calculations, the single particle states being generated from the H.F. potential using SIII force. The agreement is rather satisfactory both for the position and the strength located in the main fragment of the low-1ying 1 hg/2, $2 \mathrm{f} 7 / 2$ and $1 \mathrm{i}_{13 / 2}$ proton states in 209 Bi.

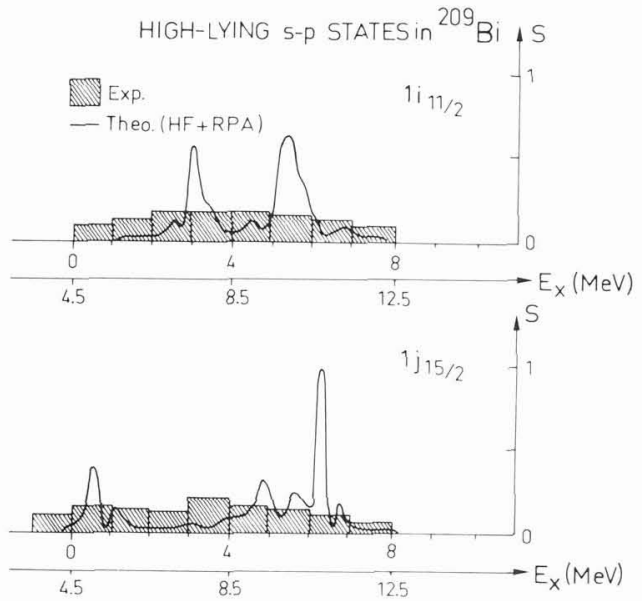

Turning now to the high-7ying 1 i $11 / 2$ and $1 \mathrm{j} 15 / 2$ strength distributions the comparison between the predicted spectral functions and the experimental ones is displayed in Fig. 17.

The damping of these high-Tying subshells is not reproduced by the calculations. The concentration of the high-lying proton strength is correctly predicted as well as the observed strong overlap of the two strength distributions. The theoretical strength functionshave much more structure than experiment, a genera? feature already noted in the case of inner-hole strength distributions $/ 26,28 /$. Surface vibrations mixing can account for $40-60 \%$ of the observed damping but the mixing with states of higher complexity is not taken into account in such calculations. 


\section{III.4 - A new excitation - The high-lying neutron particle states}

The empirical systematics on the single-particle response function include now, the inner-hole (both proton and neutron), and the proton particle states. To be complete an experimental investigation of the neutron particle states is therefore highly desirable. Most of the existing data on neutron particle strength functions come from earlier $(d, p)$ studies carried out at rather low bombarding energies or from neutron scattering experiments.

Following the transfer reaction approach, which has been quite successful in probing high-7ying proton strength distributions, we report here on the first study of quasibound or unbound high-spin neutron states using the $\left(a,{ }^{3} \mathrm{He}\right)$ on ${ }^{90} \mathrm{Zr}, 116,118,120 \mathrm{Sn}$ and ${ }^{208} \mathrm{~Pb}$ target nuclei at $183 \mathrm{MeV}$ incident energy.

The experimental method is very similar to the one already described in sect. III.1.

The $\left(\alpha,{ }^{3} \mathrm{He}\right)$ process has been chosen due to its known selectivity in angular momentum transfer for large $\&$ values. In Fig. 18 are displayed the residual energy spectra from $\left(\alpha,{ }^{3} \mathrm{He}\right)$ reaction on ${ }^{90} \mathrm{Zr},{ }^{120} \mathrm{Sn}$ and ${ }^{-208} \mathrm{~Pb}$ targets.

First let me comment on the strong selectivity of this process for high-spin single-particle states. The first low-lying neutron states in $209 \mathrm{~Pb}$ have a large spectroscopic strength $(0.8$ to 0.6$)$ but in the displayed $208 \mathrm{~Pb}\left(\alpha,{ }^{3} \mathrm{He}\right)$ ${ }^{209} \mathrm{~Pb}$ spectrum, one can see that the $1 \mathrm{j} 15 / 2$ $(\ell=7)$ state has a cross section 10 times larger than the one of the $2 g_{9 / 2}(l=4)$ groundstate. The fragmentation of the $1 j 15 / 2$ subshe 11 has been a long outstanding problem in nuclear structure. Here for the first time the fragmented components are clearly seen in the energy range 3-6 MeV.

At higher excitation energy, the observed spectrum is dominated by a wide "bump" centered around $10.7 \mathrm{MeV}$ in $209 \mathrm{~Pb}$. It may be already assumed that this large enhancement of crosssections arise from neutron stripping to highspin outer subshells. To assess such assumptions, HF calculations of the single-particle neutron spectrum in $209 \mathrm{~Pb}$ were carried out/31/ and the resulting level scheme is displayed in the left part of Fig.19. It appears that at about $10 \mathrm{MeV}$ above the $2 g_{9} / 2 \mathrm{~g} .5$. high-spin subshells namely, the $2 h_{11 / 2}, 1 k_{1} / 2$ and $1 j_{13 / 2}$ are predicted by the calculations.

In the right part of the same figure are presented the extracted angular distributions of the wel1 known low-lying $2 g_{9 / 2}, 1 i_{11 / 2}$ and 1 j $15 / 2$ neutron states together with ${ }^{\text {DWBA }}$ predictions for the indicated $n \ell j$ quantum numbers. The agreement is quite good. Therefore the experimental angular distribution for the bump located at $10.7 \mathrm{MeV}$ in ${ }^{209} \mathrm{~Pb}$ has been extracted from the data assuming for the background ?ine shape, the dashed line displayed in Fig. 18. This line shape is the result of a PWBU calculation as discussed previously in sect. III.1.1. DWBA calculations assuming a $1 j_{13 / 2}$ or a $2 h_{11 / 2}$ neutron transfer reproduces rather well the experimental cross-sections thus

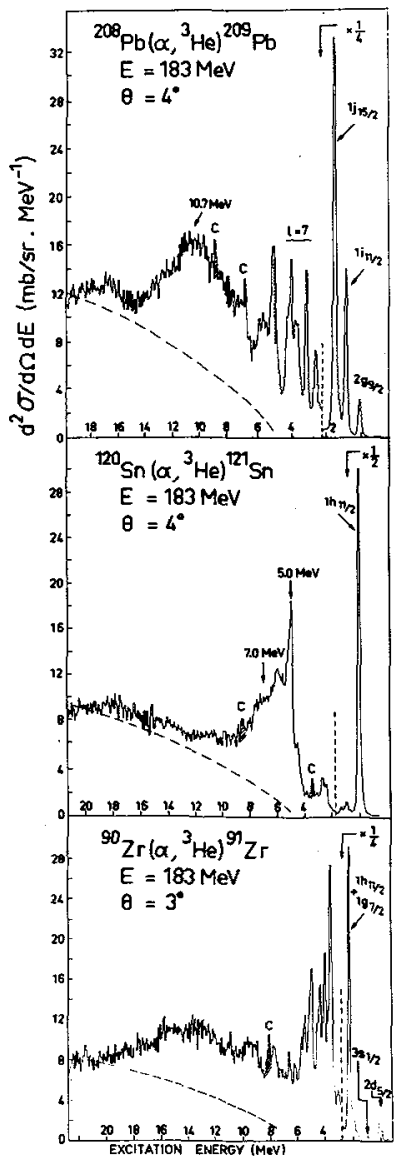

Fig. 18 - Residual energy spectra from the $\left(\alpha,{ }^{3} \mathrm{He}\right)$ reaction at $183 \mathrm{MeV}$. on ${ }^{90} \mathrm{Zr}$, $12{ }^{3} \mathrm{Sn}$ and ${ }^{208} \mathrm{~Pb}$ targets. The dashed line is the prediction of the PWBU model for the background. 
confirming our assumption that the observed enhancement is due to neutron stripping to high-spin outer subshells.

To conclude this first report on high-lying neutron states, I would like to emphasize the similarities which we observed for such highlying states in neutron pick-up or stripping experiments. As it has been pointed out in neutron pick-up studies on the tin isotopes, the 199/2 inner-hole state $(N<50)$ appears as a sharp peak with a width of less than $1 \mathrm{MeV}$ around $5 \mathrm{MeV}$ excitation energy. Moreover a shoulder at high excitation energy is always observed and corresponds to the damping of the $2 p, 1 f 5 / 2$ inner subshells. In neutron stripping experiment, one may describe the observed ${ }^{120} \mathrm{Sn}\left(\alpha,{ }^{3} \mathrm{He}\right)^{121} \mathrm{Sn}$ energy spectrum (see Fig. 18) with almost exactly the same words except that here we are populating the $1 \mathrm{hg} / 2$ and 1 i $13 / 2$ outer subshe11s $(N>82)$. Further investigations on the dependence of this observed complex structure versus neutron number in the case of the tin isotopes is underway.

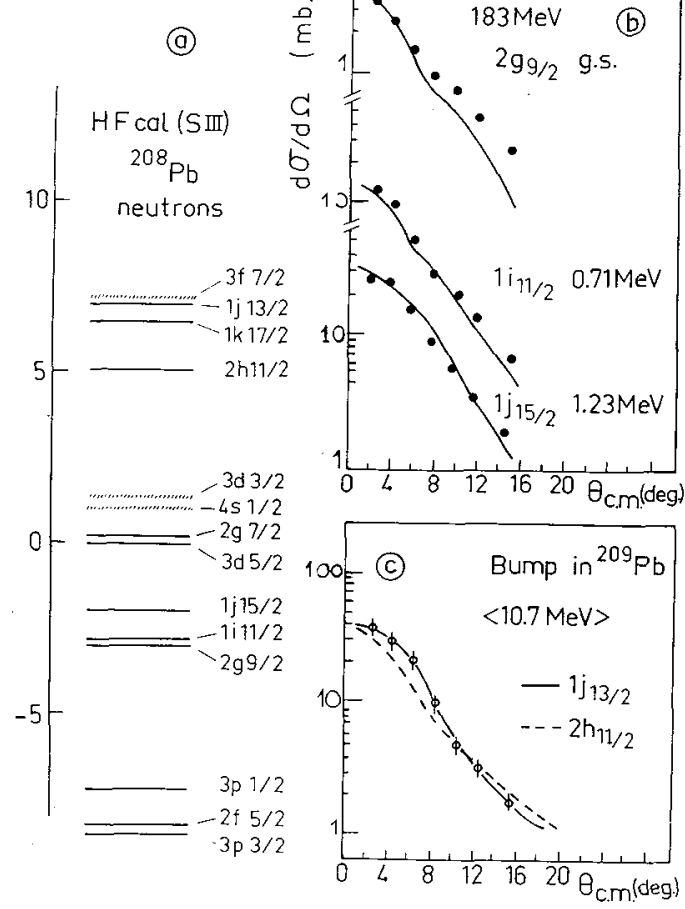

Fig.19 - a) single particle level scheme for neutron states in ${ }^{209} \mathrm{~Pb}$ (see text).

b) Experimental angular distributions and DWBA theoretical curves from the ${ }^{208} \mathrm{~Pb}\left(\alpha,{ }^{3} \mathrm{He}\right){ }^{209} \mathrm{~Pb}$ at $183 \mathrm{MeV}$ to the $1 g_{9 / 2}, 1 i_{11 / 2}$ and $1 j_{15 / 2}$ low-lying states.

c) Same as b) for the bump located at $10.7 \mathrm{MeV}$ in $209 \mathrm{~Pb}$.

\section{IV - CONCLUSIONS}

The empirical knowledge on single-particle strength functions in medium-heavy nuclei has been greatly enhanced by the observation of new high-lying modes in both proton and neutron stripping experiments. In this case the break-up of the projectile offers a natural explanation of the underlying background. At high incident energy ( $\mathrm{i}>40 \mathrm{MeV} /$ nucleon) a simple $\mathrm{plane}$ wave break-up model may be used to determine the shape and angular dependance of the observed background. Under such conditions the extraction from the experimental data of the strength distributions is more reliable. However a systematic investigation of this phenomenon is needed in order to establish firmly the method proposed here.

The theoretical approaches have reached agood qualitative understanding of the damping of such nuclear excitations. However at the quantitative level the predicted strength distributions are not enough damped (often by a factor two) as compared to the experimental results in the case of the single-particle vibrations coupling mode 1 . 
From the experimental side, one forsees the necessity of exclusive experiments, such as $\gamma$ or particle decay of high-lying states, in order to avoid background substraction problems and to gain insight on the detail of the damping mechanisms.

\section{Acknowledgments}

Much of the work reported here has been done in collaboration with a number of physicists from various laboratories and institutions.

I would like to thank my colleagues from Orsay:Drs E. Gerlic, J. Guillot, H. LangevinJoliot, S. Fortier, J.M. Maison, E. Hourani, J.P. Schapira and F. Azayes.

Drs. G. Perrin, G. Duhamel, P. Martin and V. Comparat from Grenoble,

Dr. C.P. Massolo from University of La Plata who takes a major part in the analys is of the stripping reactions while she was at Orsay, Professor G.M. Crawley from Michigan State University, Dr. D.Friesel from IUCF (USA) and Dr. B.Zwieglinski from Institute of Nuclear Research at Warsaw (Poland).

I would Tike to acknowledge Prof.V.G. Soloviev, Drs. Ch.Stoyanov, A.I. Vdovin for communications of their results prior to publication and Dr. N. Van Giai for the supply of his recent single-particle vibrations coupling calculations.

\section{REFERENCES}

11/ SAKAI M. and KUBO K., Nucl. Phys. A185 (1972) 217

12/ VAN DER WERF S.Y. et a1., Phys. Rev. Lett. 33 (1974) 712

13/ GERLIC E. et a1., Phys. Lett. 57B (1975) $33 \overline{8}$

14/ CRAWLEY G.M., Proc. of Int. Symp. on highly excited states in Nuclear Reactions, ed. by H. Ikegami and M. Muraoka, RCNP, Osaka (Japan) 1980, p.590

157 WAGNER G.T., ibid ref. 4, p.465, and contribution to this conference, p. 23.

/6/ GALES S., Proc. of Int. Conf. on Nuclear Structure, Nucl. Phys. A354 (1980) $193 \mathrm{C}$ and references herein

17/ LANGEVIN-JOLIOT H. et a 1., Phys. Lett. 114B (1982) 103

18/ SIEMSSEN R.H. et a1., Phys. Lett. 103B (1981) 323

/9/ CRAWLEY G.M. et a1., Phys. Rev. C23 (1981) 1818

$110 /$ PERRIN G. et a1., Nuct. Phys. A356 (1981) 61

$111 /$ DUHAMEL G. et ai., J. Physique G7 (1981) 1415

112 GALES S. et al., Nucl. Phys. A288 (1977) 221

113/ CRAWLEY G.M. et a 1., Phys. Rev. Lett. 39 (1977) 1457

/14/ CRAWLEY G.M. et a1., Phys. Rev. C22 (1980) 316

$115 /$ NOMURA M., Prog. Theo. Phys. 59 (1978) 1771

/16/ CRAWLEY G.M. et a1., Phys. Rev. C23 (1981) 589

117 / SOLOVIEV V.G. et aí, Nuci. Phys. A370 (1981) 13 and VORONOV V., contribution to this symposium $p .27$

118/ GERLIC E. et al., Phys. Lett. 117B (1982) 20

$/ 19 /$ KUO T.T.S. and HERLING G.H., NREL Morandum Report 2258 (1971)

120/ GALES S., ibid ref. 4, p. 425 and references herein

121/ GALES S., et a1., Phys. Rev. Lett. 48 (1982) 1593

1221 MATSUOKA'N. et al. Nucl. Phys. A311 (1978) 173

123/ WU J.R. et al., Phys. Rev. C20 (1979) 1284

124/ SHYAM R. et ai., private communication and BAUR G. et al., Helvetia Physica Acta 53 (1980) 506

/25/ SOLOVIEV V.G., STOYANOV Ch and VDOVIN A.I., Nuc?. Phys. A342 (1980) 261

126/ BORTIGNON P.F. and BROGLIA R., Nuc7. Phys. A37 (1981) $40 \overline{5}$

127/ VAN GIAI N., ibid ref. 4, p.682

128/ BERTSCH G.F., BORTIGNON P.F. and BROGLIA R.A., Rev. Mod. Physics (1983)

129/ STOYANOV Ch. and VDOVIN A.I., preprint JINR E4-83-106 (1983) to be published in Phys. Lett.

$130 /$ VAN GIAI N. and VAN THIEU Pham, to be published

/31/ VAN GIAI N., private communication. 